\title{
Integrating documentary evidence into climate reconstruction and impact studies
}

\author{
Martin Bauch', S. White', C. Camenisch ${ }^{3}$, Q. Pei ${ }^{4}$ and H. Huhtamaa ${ }^{5}$ \\ 2nd CRIAS workshop, Leipzig, Germany, 7-8 October 2019
}

PAGES' Climate Reconstruction and Impacts from the Archives of Societies working group (CRIAS; pastglobalchanges.org/crias) held its second workshop at the Leibniz Institute for the History and Culture of Eastern Europe (GWZO; pastglobalchanges.org/ calendar/2019/127-pages/1945). This was the second year of Phase 1 of the working group and demonstrated progress on the goals defined during the first workshop held in Bern, Switzerland, in October 2018 (pastglobalchanges.org/calendar/2018/127-pages/1807).

These goals were defined as:

(1) the development of methods and international comparisons in historical temperature and precipitation indices:

(2) the development of Chinese historical climatology through calibration and verification with phenological and early instrumental data; and

(3) the further integration of historical climatology results from beyond Europe, especially results from evidence not previously used for climate reconstruction.

Finally, (4) the group decided after the Bern meeting to work towards an online exhibition of historical climatological objects, in order to reach a wider public.

At the conclusion of the Leipzig meeting, participants discussed the organization of the results and ongoing research into articles for a journal special issue, which was subsequently submitted as a proposal to Climate of the Past.

Regarding the first goal, lead author D. Nash presented an advanced draft of the first global review of methods for producing temperature and precipitation indices based on written evidence. This CRIAS product will provide a foundation for comparing results across world regions and improving practices in this method for quantifying qualitative documentary evidence.
G. Adamson presented trials in the creation of indices by teams of students and experts, in order to establish the consistency of results and appropriate number required to produce valid results. S. White explained a Bayesian approach to indexing, which could draw on the wealth of historical evidence and probabilistic inferences that have necessarily been left out in current indices, and C. Camenisch examined its reconstruction skill in a sample of historical data.

\section{Q. Pei discussed ways to examine indices,} particularly the analysis of autoregression, to determine whether the statistics of index values resemble the statistics of actual seasonal and annual climate. These methodological investigations underlie research in progress and intended articles on cross-regional comparisons of extreme years for the proposed Climate of the Past special issue.

Significant progress was also made on our second goal. This CRIAS workshop represented our first cooperation with the Atmospheric Circulation Reconstructions over the Earth project (ACRE; met-acre.net) for the recovery of early instrumental data and production of high-resolution climate reanalysis.

F. Williamson from ACRE presented early instrumental data from East and Southeast Asia suitable for calibration and verification with Chinese seasonal temperature indices, and K. Lin discussed results of the Reconstructed East Asian Climate Historical Encoded Series project (REACHES; ncdc. noaa.gov/paleo-search/study/23410) on databases of Chinese historical weather records, including methods for producing regional temperature and precipitation indices.

Taken together, the re-examination of these Chinese databases and indices alongside early instrumental observations enables calibration and verification procedures that

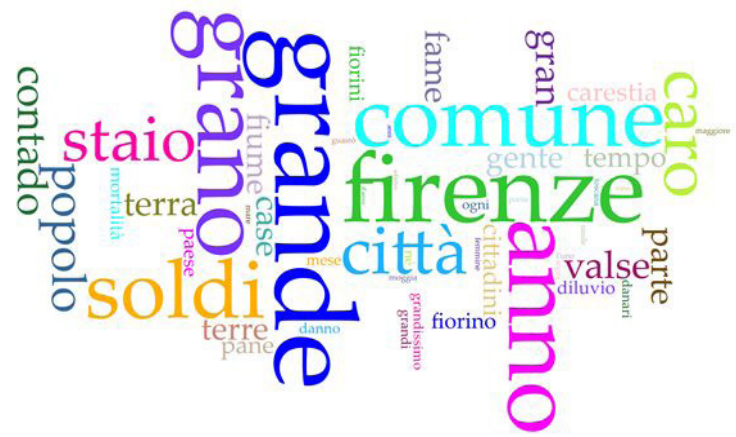

would bolster the data and enable comparisons with results from Europe and other world regions.

Regarding our third goal, S. Brönnimann provided a worldwide overview of early instrumental records in his keynote, and A.M. Burgdorf presented on a global inventory of climate reconstructions from documentary records since 1300 .

In keeping with our global and comparative focus, the meeting included further presentations of descriptive, phenological, and early instrumental evidence from China, South Asia, and Africa, as well as European documentary data (e.g. histrhone.cerege.fr). New approaches to evaluate documentary data by means of text-mining word clouds (see Fig. 1) were presented as well, analyzing semantic shifts in written records over decades and centuries.

Finally, in preparation for the workshop, a "call for objects" was issued to encourage proposals from different academic communities for objects to form a virtual "Weathered History" exhibition, which curates objects related to historical climate change for a wider public. The exhibition focuses on the themes "Remembering Disaster", "Adaption and Prevention", and "Famine and Weather".

The next CRIAS meeting will take place in Hong Kong in September 2020 (pastglobalchanges.org/ calendar/2020/127-pages/2021)

\section{ACKNOWLEDGEMENTS}

The Leipzig workshop was generously supported by PAGES, the GWZO, the Max-Planck-Institute for the Science of the Human Past (Jena, Germany), and the Heidelberg Center for the Environment (HCE).

\section{AFFILIATIONS}

'Leibniz Institute for the History and Culture of Eastern Europe, Leipzig, Germany

Department of History, Ohio State University، Columbus, USA

${ }^{3}$ Oeschger Centre for Climate Change Research and Section of Economic, Social and Environmental History, University of Bern, Switzerland

${ }^{4}$ Department of Social Sciences, Education University of Hong Kong, China

'Heidelberg Center for the Environment, Heidelberg University, Germany

CONTACT

Martin Bauch: martin.bauch@leibniz-gwzo.de

Figure 1: A word cloud created with Voyant Tools based on all reports on weather extremes and food prices/

crisis in Giovanni Villani's Nuova Cronica published in the 14th century. 